\title{
Publisher's Note: Examining evolving performance on the Force Concept Inventory using factor analysis [Phys. Rev. Phys. Educ. Res. 13, 010103 (2017)]
}

M. R. Semak, R. D. Dietz, R. H. Pearson, and C. W. Willis (Received 19 January 2017; published 3 February 2017)

DOI: 10.1103/PhysRevPhysEducRes.13.019903

This paper was published online on 10 January 2017 with an incorrect volume number in the article identifier. The correct volume is "13." The paper has been corrected as of 19 January 2017. 